\title{
Sistema de riego basado en controlador PID para la adecuación de la humedad del suelo en invernaderos
}

\section{Irrigation system based on PID controller for the adaptation of soil moisture in greenhouses}

\author{
Sallelis Calderón ${ }^{l}$, Miriam Centella ${ }^{1}$, Melanie Samaniego ${ }^{l}$, Edwin Collado $^{1,2 *}$ \\ ${ }^{I}$ Centro Regional de Azuero, Universidad Tecnológica de Panamá \\ ${ }^{2}$ Centro de Estudios Multidisciplinarios en Ciencias, Ingeniería y Tecnología-AIP (CEMCIT-AIP)
}

\begin{abstract}
Resumen Actualmente el sector agrícola es el mayor consumidor de agua a nivel mundial. Esto se debe a que la mayoría de los agricultores utilizan técnicas de riego tradicionales, logrando así una mala utilización del agua en sus cultivos. Por ello, en los últimos años ha crecido el interés por desarrollar soluciones tecnológicas que permitan controlar el consumo de agua en este sector. El uso de estas soluciones en invernaderos ha mejorado aún más el consumo de agua, ya que estas estructuras permiten controlar mejor variables como la humedad de suelo. En este artículo se propone un sistema de control de riego que toma en cuenta las propiedades del cultivo y la humedad del suelo dentro del invernadero para decidir el momento adecuado del riego. Este diseño cuenta con un controlador proporcional, integral y derivativo (PID), un sistema de aspersores y una red de sensores. Las simulaciones fueron realizadas en LabVIEW, que es un software para la simulación, modelado para el análisis de sistemas que requieren pruebas, mediciones y control. Este software nos permite variar los parámetros del controlador PID para observar el comportamiento de la humedad en el suelo mediante una interfaz gráfica. De acuerdo con nuestros resultados, la implementación de este sistema de control de riego permite disminuir el consumo de agua en el sector agrícola, mientras se logra un buen desarrollo de las plantas en el sistema.
\end{abstract}

Palabras clave Control de humedad, controlador PID, invernadero, sistema de control y sistema de riego.

\begin{abstract}
Currently the agricultural sector is the largest consumer of water worldwide. This is because most farmers use traditional irrigation techniques, thus achieving poor water use in their crops. For this reason, in recent years interest has grown in developing technological solutions that allow controlling water consumption in this sector. The use of these solutions in greenhouses has further improved water consumption, since these structures allow better control of variables such as soil moisture. In this article, an irrigation control system is proposed that considers the properties of the crop and the humidity of the soil inside the greenhouse to decide the right time to water. This design features a proportional, integral, and derivative (PID) controller, a sprinkler system, and a sensor network. The simulations were performed in LabVIEW, which is software for simulation, modeling, and analysis of systems that require testing, measurement, and control. This software allows us to vary the parameters of the PID controller to observe the behavior of moisture in the soil through a graphical interface. According to our results, the implementation of this irrigation control system allows reducing water consumption in the agricultural sector, while achieving a good development of the plants in the system.
\end{abstract}

Keywords Humidity control, PID controller, greenhouse, control system, irrigation system.

* Autor de correspondencia: edwin.collado@utp.ac.pa

\section{Introducción}

El agua es un recurso natural finito, renovable y vulnerable, el cual es indispensable para el bienestar de las personas, el funcionamiento de los ecosistemas y el desarrollo de la economía [1]. A pesar de que la superficie de nuestro planeta está cubierta en un $70 \%$ de agua, este es un recurso escaso porque el porcentaje aprovechable es cerca del $0.7 \%$ del volumen total [2]. Hoy en día, Panamá se encuentra como cuarto país en el mundo en consumo de agua y el primero en América Latina, debido principalmente al mal manejo del recurso [3]. En países en vía de desarrollo como Panamá, donde la agricultura es un sector clave en las actividades económicas de su población. Sin embargo, el sector agrícola es el mayor consumidor de agua en el país, no solo por el crecimiento de sus actividades, sino porque la mayoría de los agricultores no cuentan con sistemas de riego eficientes y controlados para reducir el consumo desmesurado del agua [4]. Por ejemplo, alrededor del $43 \%$ de las extracciones de agua dulce que se realizan en el país son para uso agrícola. A pesar de eso, existe escasez de agua en ciertas zonas y épocas del año, especialmente en la región de Arco Seco [3]. Esto podría 
dar paso a nuevas crisis en otros sectores como lo son la alimentación, saneamiento y salud.

Es un recurso limitado, el uso eficiente del agua en sistemas de riego en cultivos, debe ser el principal objetivo para reducir el consumo de los recursos hídricos a nivel mundial. Por ello, el desarrollo de soluciones tecnológicas para controlar el consumo de agua en sistemas de riego resulta conveniente [4],[5]. Por otra parte, con estas herramientas los cultivos obtienen mayor rendimiento y mejor calidad de sus productos finales, ya que obtienen la cantidad ideal de agua [6]. El riego aplicado en el momento adecuado y la cantidad realmente necesaria son factores indispensables para obtener un cultivo con un buen rendimiento. El exceso de agua arrastra los nutrientes del suelo hasta una profundidad fuera del alcance de las raíces y a su vez desplaza el aire existente en el suelo el cual provoca que estas carezcan de oxígeno [7].

Un sistema de riego controlado permite saber, mediante la medición de la humedad en el suelo, cuándo y qué tanto regar, asegurando que se cumplan las necesidades hídricas de las plantas según los requerimientos del tipo de suelo utilizado y del cultivo. Esto ha llevado a gran parte de la comunidad científica a desarrollar soluciones tecnológicas que ayuden a reducir el consumo de agua en el sector agropecuario. Por ejemplo, en [8] presentan un sistema de riego inteligente de bajo costo que controla la cantidad y dirección del flujo de agua, utilizando información de una red de sensores de humedad de suelo. El trabajo presentado en [9] propone un sistema para monitorear continuamente el nivel de humedad del suelo, para decidir el tiempo y cantidad de agua necesaria en el cultivo. Se realizaron pruebas con diferentes tipos de suelo y diferentes niveles de sequedad, donde observaron que los suelos arenosos requieren menos agua que los suelos arcillosos y estos requieren la mayor cantidad de agua para el riego. Los autores en [10] desarrollaron un sistema que controla la cantidad de agua y realiza el proceso de forma automática, utilizando un arreglo de sensores y servicio de nube en Internet mediante módulos GSM/GPRS. Los resultados obtenidos demuestran que el sistema logra controlar de manera eficaz el consumo de agua.

En países de Latinoamérica, se han realizado estudios para resolver la problemática de escasez de agua, mejorando el consumo y reduciendo el desperdicio. Por ejemplo, en Argentina, se ha realizado un estudio en donde se implementaron Redes Inalámbricas de Sensores (RIS) para monitorear en tiempo real la humedad del suelo, y así aplicar mecanismos para la administración del agua. Los datos obtenidos pueden ser analizados y utilizados en el manejo de agua en los cultivos [11]. Además, en este país se han desarrollado otros sistemas de riego automatizado con sensores capacitados para controlar el uso del agua, logrando un ahorro en el consumo y una mejora en la calidad en los productos finales [4]. En un estudio similar en Perú, se diseñó un sistema automatizado de riego para césped de jardín, utilizando sensores de humedad por medio de la tecnología de Reflectometría de Dominios de Frecuencia (FDR). Este puede ser implementado para varias condiciones climáticas, de cualquier tipo de suelo y cultivo, con el objetivo de indicar en qué momento iniciar y terminar el riego [12]. En Panamá se han propuesto algoritmos de control para resolver los problemas del consumo de agua, tomando en cuenta las características de las plantas y el ambiente que las rodea. Este proyecto compara el sistema propuesto con sistemas de riego de suministro fijo de agua, en donde se proporcionó una solución óptima para reducir el consumo de agua [13], [14].

Debido a las ventajas del control en los sistemas de riego, este trabajo propone un método inteligente para el control de la humedad del suelo en invernaderos. El objetivo de este sistema es disminuir el uso de agua al administrar la cantidad y las veces necesarias para cumplir con las necesidades hídricas de las plantas, manteniendo la humedad del suelo dentro del rango adecuado para evitar enfermedades en el cultivo. Para el funcionamiento del sistema, se utiliza un controlador proporcional, integral y derivativo (PID), que es uno de los controladores más empleados y versátiles del mercado. La mayor ventaja del sistema propuesto es el control de tiempo de respuesta, porque el controlador PID permite disminuir y lograr que el sistema responda más rápido. De igual manera, nos permite aumentar la precisión de la respuesta, mediante la configuración de los parámetros de control y a la vez hacer las correcciones del funcionamiento [15].

El artículo está organizado de la siguiente manera: La sección 2 describe el diseño y la metodología utilizada en el desarrollo del sistema propuesto. La sección 3 presenta los resultados preliminares obtenidos. La sección 4 presenta la discusión y conclusiones de los resultados de la investigación.

\section{Diseño y Metodología}

El análisis de este proyecto está centrado en una investigación aplicada, con el objetivo de presentar una posible solución a un problema existente en el sector agrícola, desarrollando un algoritmo y detallando los componentes necesarios para la construcción e implementación de este sistema.

Para el inicio de este proyecto se utilizaron las mediciones tomadas por el sistema de monitoreo de humedad de suelo del proyecto de investigación SENACYT APY-NI-2018-09. Estos datos fueron recopilados en el programa Excel para ser utilizados para el Programa de Diseño y Simulaciones de Sistemas LabVIEW de National Instruments.

En este artículo se presentará la formulación del problema, el modelo conceptual del sistema, que incluye un diagrama de flujo que indica el funcionamiento, la simulación y los resultados.

\subsection{Formulación del problema}

La humedad de suelo es una de las variables más importantes en el desarrollo de los cultivos en los invernaderos, por lo tanto, es necesario un mecanismo de control en sistemas de riego para mantener el nivel adecuado de humedad. 
En los últimos años, los agricultores se han preocupado por disminuir el consumo de agua en sus cultivos, mano de obra, recursos económicos, uso de plaguicidas; los cuales son productos de métodos tradicionales de riego. Por esta razón, se ha propuesto en este trabajo un sistema automatizado basado en controlador PID que controle el riego según las propiedades del cultivo y los requerimientos del productor.

\subsection{Modelo conceptual}

Esta sección se presenta el sistema de control de humedad de suelo propuesto. Además, discute sus componentes y el funcionamiento.

El diseño del sistema automatizado de riego consta de sensores de humedad de suelo para medir la variable en todo el terreno de producción. Estos sensores están conectados mediante una red de comunicación inalámbrica con un controlador central PID, que se encarga de enviar acciones a los actuadores del sistema para obtener la humedad adecuada. Este controlador cuenta con tres acciones: parámetro proporcional, que es el que intenta minimizar el error del sistema y aplica una acción dependiendo que tan lejos esté el valor medido al valor de referencia; el parámetro integral, que almacena los errores pasados con el fin de reducir el error en régimen permanente y el parámetro derivativo que es el encargado de la velocidad de respuesta del sistema [16]. En la figura 1, se muestra el Modelo Conceptual del Sistema propuesto.

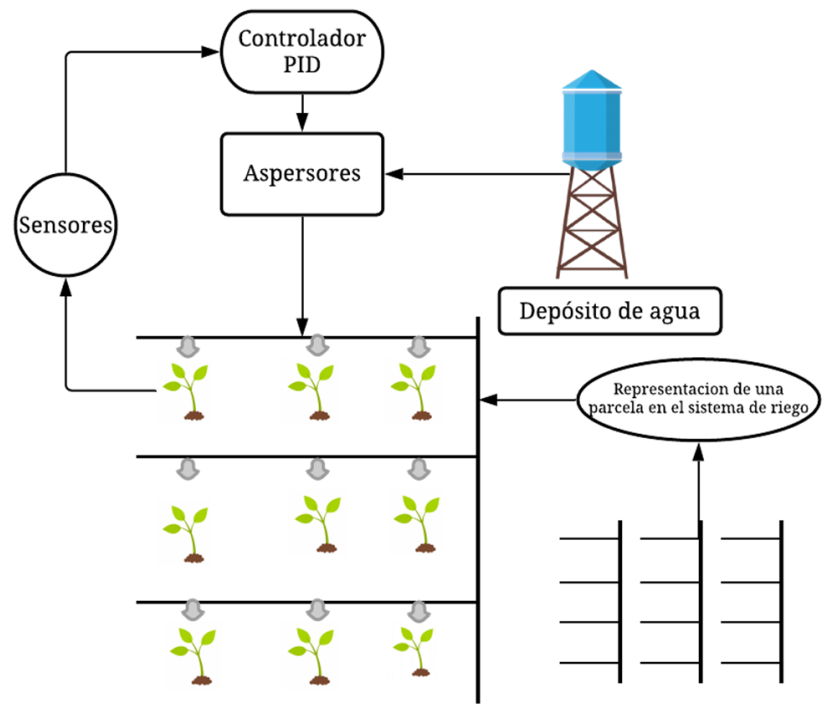

Figura 1. Modelo conceptual del sistema de control de riego.

En la figura 2, se muestra un Diagrama de Flujo que presenta el funcionamiento del sistema. Después de seleccionar el valor de referencia (humedad adecuada), es comparado con los valores medidos por los sensores. La diferencia entre el valor de referencia y el estado actual del sistema genera una señal de error que es enviada a la entrada del controlador PID, que es el encargado de mantener la variable dentro del rango deseado. Luego, el controlador verifica si el valor obtenido es menor o mayor que el valor de referencia para así tomar las decisiones correspondientes en el actuador. Esta acción consiste en encender y/o apagar los aspersores del sistema de riego en el invernadero.

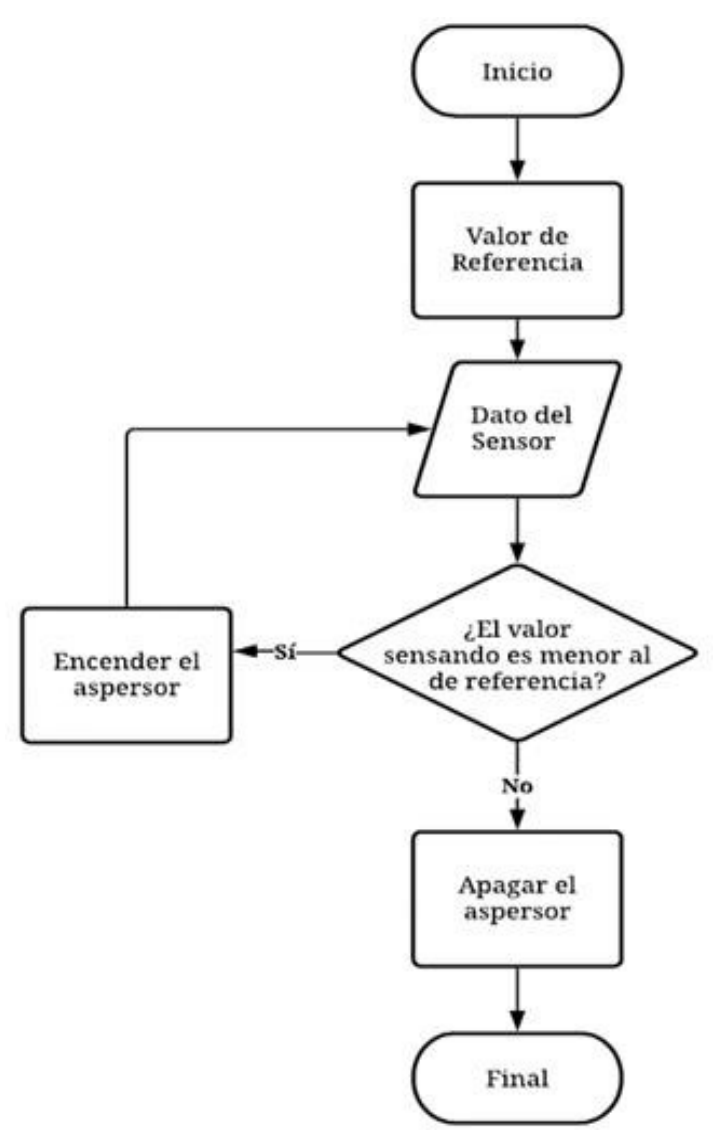

Figura 2. Diagrama de flujo del funcionamiento del sistema de control de humedad.

\subsection{Materiales}

Para desarrollar este proyecto de manera tangible se necesitan ciertos elementos indispensables, los cuales detallaremos en la siguiente tabla.

Tabla 1. Materiales y costo aproximado de implementación

\begin{tabular}{|c|r|}
\hline Material & $\begin{array}{c}\text { Costo } \\
\text { aproximado }\end{array}$ \\
\hline Controlador PID & $\mathrm{B} / .64 .99$ \\
\hline Sensor de humedad de suelo & $\mathrm{B} / .245 .00$ \\
\hline Micro aspersor & $\mathrm{B} / .21 .00$ \\
\hline Tuberías de PVC & $\mathrm{B} / .5 .99$ \\
\hline Rollo de manguera PVC & $\mathrm{B} / .26 .50$ \\
\hline Total & $\mathrm{B} / . \mathbf{3 6 3 . 4 8}$ \\
\hline
\end{tabular}


Los precios especificados en la tabla son unitarios y las cantidades dependerán del diseño del invernadero en el que se vaya a aplicar este sistema de control. Es importante mencionar que el costo de los sensores puede variar según el nivel de efectividad que se desee y los requerimientos del productor. Para este proyecto, se consideran sensores de humedad de suelo con una alta exactitud de medición y mayor rango de alcance.

\section{Simulación y resultados}

En la simulación de nuestro proyecto utilizamos el programa LabVIEW de National Instruments, el cual es un Software de Ingeniería para el desarrollo de sistemas que requieren pruebas, medidas y control. Este software consiste en una interfaz de programación gráfica y es utilizado para simulaciones en tiempo real. Este programa nos permite utilizar instrumentos virtuales (VI's) para imitar equipos y componentes reales [17].

Dentro de la simulación se utilizaron tres estructuras de programación: ciclo while, ciclo for y una estructura de casos, cada una de ellas con funciones diferentes dentro del sistema. Con la ayuda del VI Read Delimited Spreadsheet, se extrajo una lista de datos que corresponden a los valores medidos de los sensores del sistema de monitoreo, los cuales fueron organizados matricialmente para que puedan ser interpretados por LabVIEW. Estos datos representan la variable de proceso que será continuamente comparada con el punto de referencia (setpoint). Como mencionamos anteriormente, esta comparación resulta en una señal de error que entrará al controlador PID y le indicará cual es el error que existe entre el valor deseado y el valor tomado por el sensor. LabVIEW nos ofrece un VI de controlador PID, el cual nos permite ingresarle los valores de ganancia proporcional, así como el tiempo integral, derivativo y observar el comportamiento del sistema controlado. Se experimentó con diferentes valores de ganancia proporcional y observando el comportamiento del sistema para los cultivos seleccionados, se determinó un valor de ganancia y el sistema presenta una mejor respuesta. De igual manera, se experimentó con el tiempo integral y el tiempo derivativo, observando que el sistema no presente irregularidades. En esta parte, se experimentó con el tiempo integral y se observó una mejora con un tiempo de $0.010 \mathrm{~min}$ $(0.6 \mathrm{~s})$. Para el tiempo derivativo, se realizó el mismo proceso en donde el tiempo de $0.005 \mathrm{~min}(0.3 \mathrm{~s})$ es el tiempo máximo para el cual el sistema se comporta adecuadamente. Finalmente, se determinó que para una ganancia de 25 el sistema tenía una mejor respuesta, por lo que se decidió asignar este valor.

Con la finalidad de tener la mayor información del sistema que se está controlando, se optó por determinar la diferencia de humedad necesaria para llegar al valor de referencia. Se hizo uso de una estructura de caso, de manera que para humedades donde se necesite riego y se pueda saber el porcentaje de humedad faltante. En figura 3, se puede observar cómo queda estructurado el sistema de control de riego en la pantalla de simulación de LabVIEW.

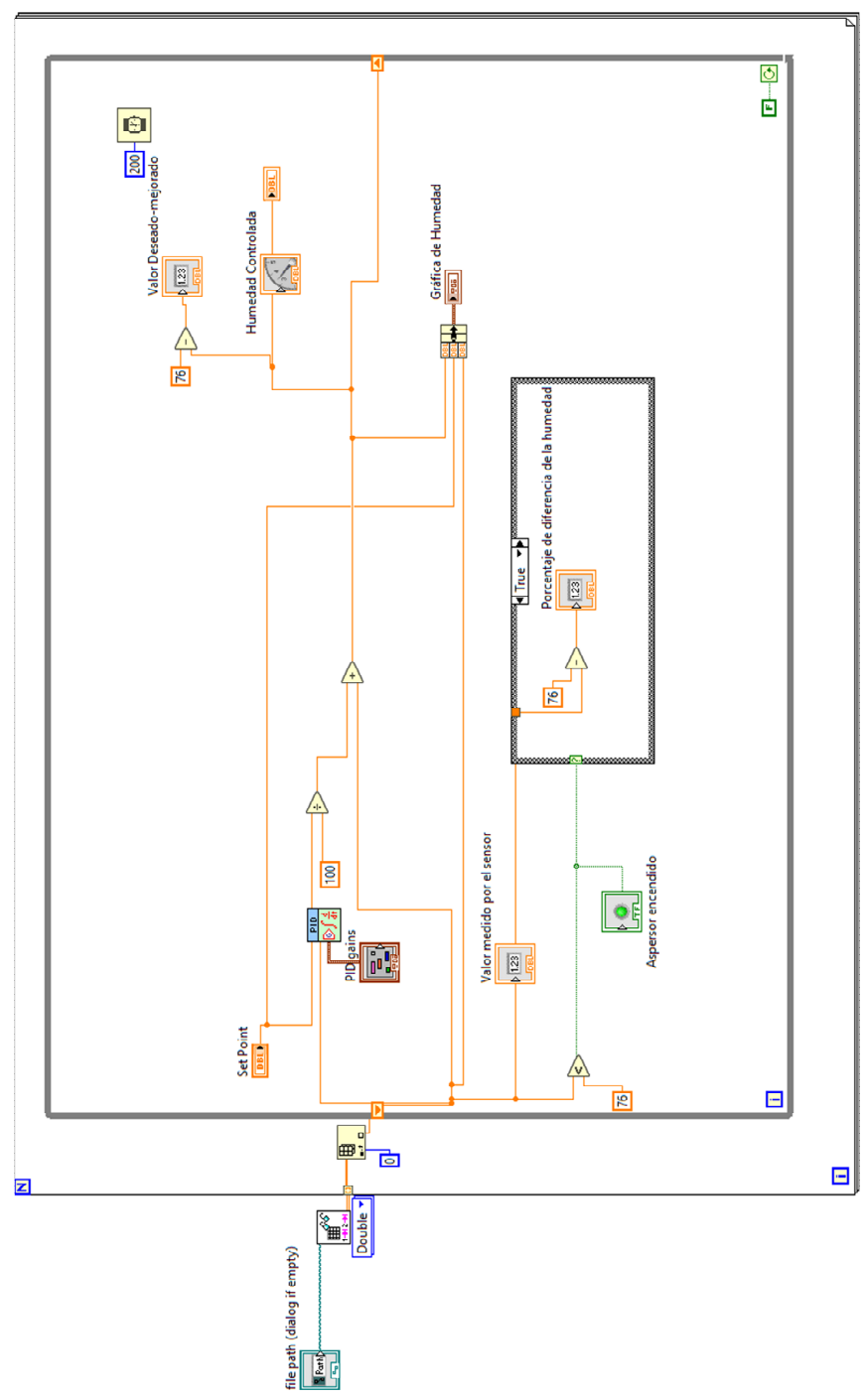

Figura 3. Simulación del sistema de control en LabVIEW.

Los datos de humedad de suelo fueron sensados por dos semanas cada media hora, de los cuales solo se estudió una fracción de datos que representaban mayores variaciones. Sin embargo, para observar el funcionamiento, se utilizó un tiempo de repetición del ciclo de un segundo, el cual hace el papel de tiempo de muestreo.

Para observar el funcionamiento del sistema se seleccionaron dos cultivos comunes en Panamá: el ñame y la lechuga. Según la información consultada, el cultivo de ñame necesita que la humedad del suelo oscile entre $76 \%$ y $83 \%$ para un buen desarrollo [18]. Por ello, en la simulación se tomó un punto de referencia (setpoint) de $76 \%$ de humedad de suelo.

Después de realizar la simulación, LabVIEW nos permite observar el cambio en las mediciones de humedad, tomando en 
cuenta que la humedad adecuada para el desarrollo de un cultivo de ñame es del 76\%. En figura 4, se puede visualizar la diferencia entre el valor medido por el sistema de monitoreo y el mejorado gracias al sistema de control de humedad en el suelo propuesto, en donde la línea de color rojo representa el valor de referencia, la verde es la humedad medida por el sensor y la blanca representa la humedad modificada.

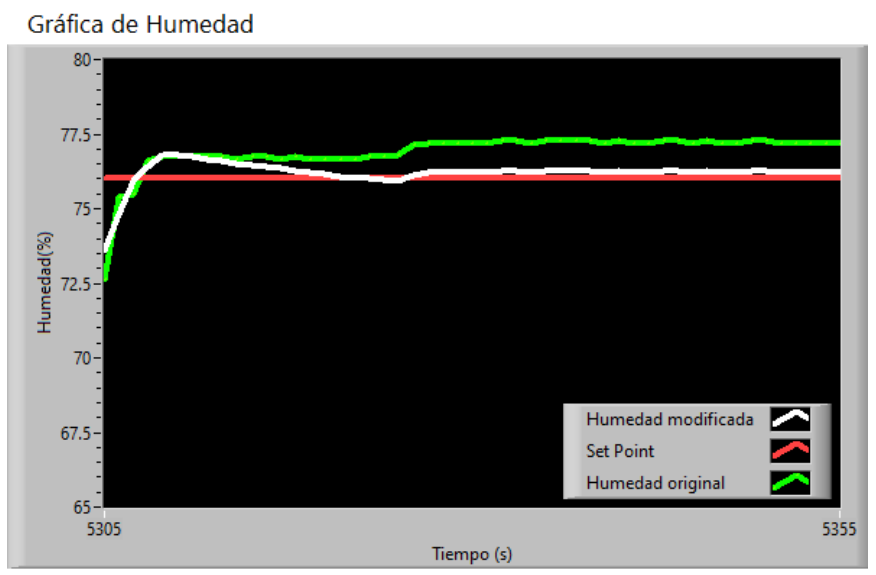

Figura 4. Gráfica del comportamiento de la humedad del suelo para el ñame.

Analizada la gráfica, podemos notar que el controlador ha mejorado la humedad medida por el sistema de monitoreo y que el valor controlado converge al valor de referencia de humedad de suelo a medida que transcurre el tiempo.

LabVIEW, mediante indicadores, nos muestra el valor de humedad controlado para cada valor medido por el sistema de monitoreo. De esta manera, podemos calcular que tan lejos están ambos valores con el de referencia y así comprobar lo observado en la gráfica.

En las siguientes operaciones, podemos observar la diferencia entre la humedad medida $\left(H_{m}\right)$ y el valor de referencia $\left(H_{r}\right)$ (ver ecuación 1) y la diferencia entre el valor de humedad controlado $\left(H_{c}\right)$ y el de referencia $\left(H_{r}\right)$ (ver ecuación 2).

$$
\begin{aligned}
& H_{m}-H_{r}=77.07 \%-76.00 \%=1.07 \% \\
& H_{c}-H_{r}=76.07 \%-76.00 \%=0.07 \%
\end{aligned}
$$

Si se comparan los resultados obtenidos de nuestro sistema de control con los del sistema de monitoreo, podemos observar que existe una menor diferencia entre el valor controlado y el valor de referencia, tal como se observa en los resultados de la figura 4.

Según estudios realizados, el cultivo de lechuga necesita una humedad en el suelo que oscile entre $60 \%$ y $80 \%$ para que tenga un buen desarrollo [19]. Por ello, se utilizó un punto de referencia de $73 \%$ de humedad de suelo para la simulación. En la siguiente figura, se puede prestar atención al comportamiento de la humedad de suelo utilizando LabVIEW.

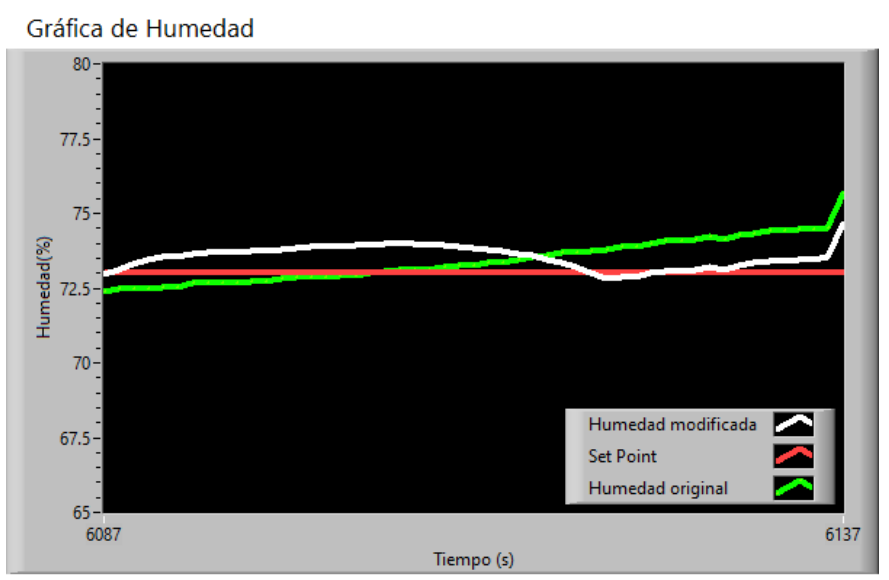

Figura 5. Gráfica de comportamiento de la humedad del suelo para la lechuga.

En esta figura la línea roja representa el valor de referencia, la verde la humedad medida por el sistema de monitoreo y la blanca la humedad controlada por el sistema propuesto. Observando la gráfica para el cultivo de lechuga, se puede notar que la humedad controlada está más cerca del valor de referencia (setpoint) que la humedad medida por el sensor.

Mediante los indicadores de LabVIEW, podemos obtener el valor de humedad controlada correspondiente a cada valor de humedad medida, los cuales nos permiten en las siguientes operaciones (3) y (4) comprobar lo observado en la gráfica.

$$
\begin{aligned}
& H_{m}-H_{r}=74.13 \%-73.00 \%=1.13 \% \\
& H_{c}-H_{r}=73.13 \%-73.00 \%=0.13 \%
\end{aligned}
$$

Podemos, prestar atención nuevamente que existe una menor diferencia entre el valor controlado y el valor de referencia, tal como se observa en los resultados de la figura 5.

La figura 6, muestra la herramienta final que se le brinda al productor, donde se puede observar el comportamiento del sistema de control en tiempo real y recibir alarmas. Cuenta con LED indicadores que permiten verificar si el sistema de riego por aspersores está encendido o apagado. De igualmente, el indicador numéricos permite visualizar en tiempo real los valores medidos, controlados y deseados, así como el porcentaje de diferencia entre ellos. Este sistema cuenta con interfaz para ajustar los parámetros del controlador PID: los valores de ganancia proporcional, tiempo integral y derivativo.

Al ser un sistema programable, el productor tiene la libertad de personalizar la herramienta según los requerimientos de su sistema de producción. Esto permite que el sistema de control sea adaptable a todo tipo de productor: pequeño, mediano y grande. 


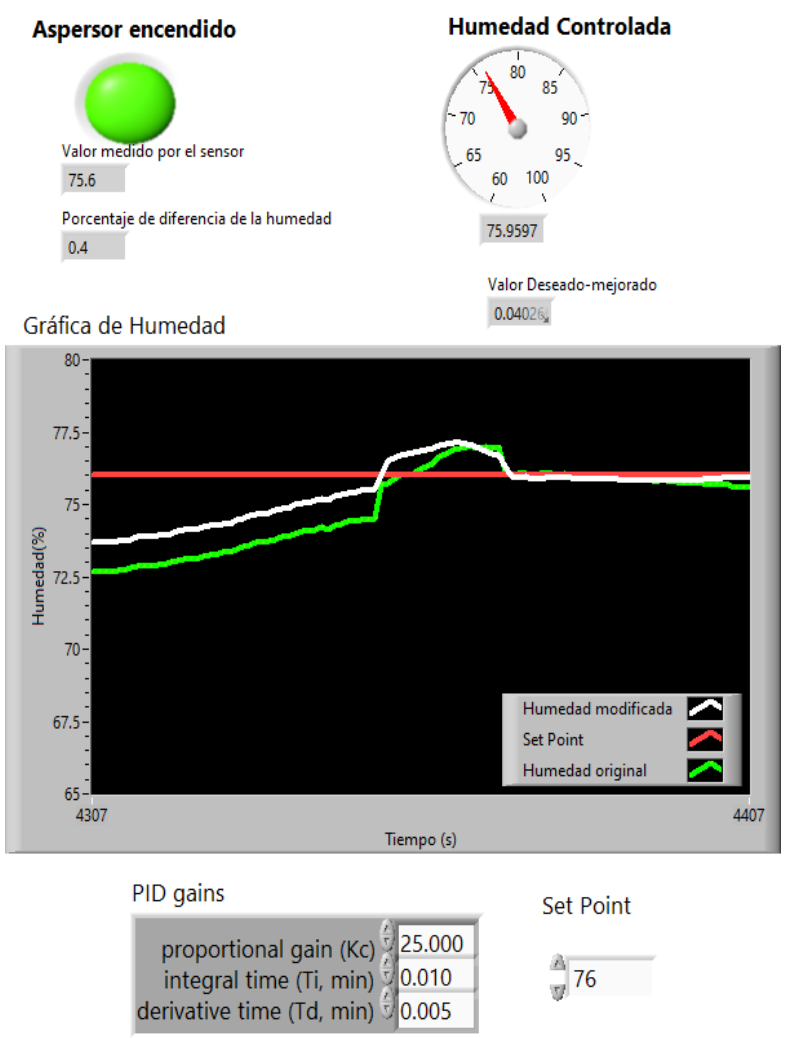

Figura 6. Diseño de interfaz de control de riego en LabVIEW.

\section{Discusión y conclusiones}

El uso de sistemas de control de riego nos permite mejorar el desarrollo de los cultivos al utilizar optativamente la cantidad de agua necesaria para la planta, mientras se reduce su consumo. Esto se logra al determinar el momento oportuno para aplicar el riego gracias al conocimiento de la humedad del suelo y la humedad que necesita el cultivo.

El Sistema de Control propuesto en este trabajo utiliza controladores PID, los cuales son utilizados comúnmente en este tipo de proyecto, debido a su versatilidad en sistemas de control y su facilidad para ajustar los valores de ganancia adecuados para llegar a una respuesta deseada en el sistema, mediante sus parámetros de control. Una gran ventaja de este sistema es que puede ser utilizado para cualquier tipo de cultivo, garantizando en todo momento el nivel adecuado de humedad de suelo. Los resultados obtenidos demuestran que el controlador ha mejorado el consumo de agua en el sistema de riego, alcanzando una diferencia mínima entre el valor modificado y el valor óptimo de humedad deseado. Así, se observa que el valor controlado de humedad converge al valor deseado a medida que pasa el tiempo, lo cual garantiza un valor óptimo constante una vez se obtenga el nivel de humedad requerido. Para llegar al comportamiento esperado, es importante colocar los valores de ganancia y de tiempos adecuados, de lo contrario el sistema puede presentar cambios irregulares.
En trabajos futuros se puede estudiar el modelo matemático de este sistema de manera que se pueda evaluar los parámetros del controlador PID y lograr que su comportamiento sea óptimo. Se espera que en estas investigaciones se pueda determinar la cantidad de agua exacta que requiere el cultivo para tener una humedad adecuada.

\section{AGRADECIMIENTOS}

Agradecemos al Dr. Edwin Collado, por facilitarnos los datos de las mediciones de humedad en el suelo, los cuales fueron imprescindibles para el desarrollo de nuestro trabajo de investigación. Estos datos fueron obtenidos en su proyecto de investigación APY-NI-2018-09, financiado por SENACYT. El Dr. Collado, agradece al Sistema Nacional de Investigación (SNI) y a la SENACYT por brindar el apoyo requerido en la realización de sus investigaciones.

\section{REFERENCIAS}

[1] J. Díaz. "El Agua en Cuba: un desafío a la sostenibilidad", Ingeniería Hidráulica y Ambiental, vol. 39 No.2. Mayo-agosto 2018.

[2] O. Rey Santos. "El Agua en tiempos de crisis" Revista de Estudios Estratégico, No.1, enero-junio 2014.

[3] C. Garcimartín. J. Astudillo, O. Garzonio. "El Agua en la Economía de Panamá”. Banco Interamericano de Desarrollo, Abr. 2020.

[4] C. Schugurensky, F. Capraro. "Control Automático de Riego Agrícola con Sensores Capacitivos de Humedad de Suelo. Aplicaciones en Vid y Olivo". Instituto de Automática, Universidad San Juan, Argentina. Dic. 2013.

[5] E. Collado, A. Fossatti, and Y. Saez. "Smart farming: A potential solution towards a modern and sustainable agriculture in Panama.” AIMS Agriculture and Food 4.2, pp 266-284, 2018.

[6] H. Avilés. "El Valor del Agua en la Agricultura", La Granja, 2006

[7] J. Bareiro "El Agua y las Plantas", ABC Rural, 2005. [Online] Available: impresa/suplementos/abc- rural/el-agua-y-las-plantas811751.html

[8] Sahu, C. Kumar and P. Behera. "A low-cost smart irrigation control system." 2015 2nd International conference on electronics and communication systems (ICECS). IEEE, 2015.

[9] Agbetuyi, Ayoade F., et al. "Design and implementation of an automatic irrigation system based on monitoring soil moisture." Journal of Electrical Engineering, 2017.

[10] Avşar, Ercan, et al. "Development of a cloud-based automatic irrigation system: A case study on strawberry cultivation." 2018 7th International Conference on Modern Circuits and Systems Technologies (MOCAST). IEEE, 2018.

[11] M. Flores, F. Flores, V, Velasco, G. González. "Monitoreo de humedad en suelo a través de red inalámbrica de sensores". Tecnología y Ciencias del Agua. Vol.6 No. 5, Jiutepec. Sept-oct 2015.

[12] Z. Varas "Diseño de un sistema electrónico para riego de césped de jardín empleando un sensor de humedad de suelo y comunicación inalámbrica”. Tesis de Grado. Universidad Católica del Perú. Lima. Abr. 2013.

[13] E. Collado, Y, Sáez. "Sistema de riego inteligente para optimizar el consume de agua en cultivos en Panamá”.15 ${ }^{\text {th }}$ LACCEI 
International Multi-Conference for Engineering, Education, and Technology. Jul. 2017.

[14] E. Collado, Y, Sáez. "Optimization of Irrigation Time Programming to Reduce Water Consumption in Crops". 17th LACCEI International Multi-Conference for Engineering, Education, and Technology. Jul. 2019.

[15] A. Rubio "Ventajas de los reguladores PID". Instrumentación Digital. [Online] Available: https://www.instrumentaciondigital.es/4-ventajas-de-losreguladores-pid/ . 2019

[16] C. Pardo "Controlador PID". Available: https://www.picuino.com/es/arduprog/control-pid.html

[17] National Instruments. LabVIEW.

Available: https://www.ni.com/es-cr/shop/labview.html

[18] M. Ávila "Manejo Integrado del Cultivo del Name Espino". Cultivo de Name en Colombia. Dic. 2013.

[19] R. González, C. Duarte, L. Montero. "Influencia de diferentes niveles de humedad del suelo en el cultivo de la lechuga en condiciones de organopónico en La Habana". Ingeniería Agrícola. Vol. 2, No. 2, pp 51-55. Jul-dic 2012. 